\title{
S-matrix singularities and CFT correlation functions
}

\author{
Carlos Cardona ${ }^{a}$ and Yu-tin Huang ${ }^{a, b}$ \\ ${ }^{a}$ Physics Division, National Center for Theoretical Sciences, National Tsing-Hua University, \\ No.101, section 2, Kuang-Fu Road, Hsinchu, Taiwan \\ ${ }^{b}$ Department of Physics and Astronomy, National Taiwan University, \\ Taipei 10617, Taiwan \\ E-mail: cargicar@gmail.com, yutinyt@gmail.com
}

ABSTRACT: In this note, we explore the correspondence between four-dimensional flat space S-matrix and two-dimensional CFT proposed by Pasterski et al. We demonstrate that the factorisation singularities of an $n$-point cubic diagram reproduces the AdS Witten diagrams if mass conservation is imposed at each vertex. Such configuration arises naturally if we consider the 4-dimensional S-matrix as a compactified massless 5-dimensional theory. This identification allows us to rewrite the massless S-matrix in the CHY formulation, where the factorisation singularities are re-interpreted as factorisation limits of a Riemann sphere. In this light, the map is recast into a form of $2 d / 2 d$ correspondence.

Keywords: Conformal Field Theory, Scattering Amplitudes

ARXIV EPRINT: 1702.03283 


\section{Contents}

1 Introduction 1

2 The PSS proposal 3

2.1 The three point contact term 5

3 Contact diagrams from factorization singularities $\quad 6$

3.1 A four-point example 6

3.2 The $n$-point generalization 9

$\begin{array}{lll}4 & \text { A } 2 d-2 d \text { duality } & 13\end{array}$

5 Massless singularities $\quad 14$

6 Conclusions and outlook $\quad 16$

\section{Introduction}

The isomorphism between the Lorentz group in four dimensions and the Mobius group of conformal transformations in two dimensions, have been used in several theoretical approaches for the computation of scattering amplitudes in the past. It is indeed the corner stone of the realisation of Penrose's Twistor Space [1] and the impressive subsequent development of scattering amplitudes of massless particles in Twistor space, started by Witten more than a decade ago [2]. A generalisation of this isomorphism is the Embedding Formalism for the $d$-dimensional conformal group, built upon the work of Dirac [3] and which has been particularly useful in the context of the AdS/CFT correspondence

Recently, there has been a growing effort in writing the dynamics of four-dimensional Minkowski space in terms of observables in two-dimensional conformal field theory, greatly motivated by the renewed interest on the asymptotic BMS symmetries in gravitational theories $[4,5]$ (for a more recent discussion see [6]), based on the observation that the Lorentz group in four-dimension acts as the Mobius group on the two dimensional nullinfinity boundary, recently baptised as the celestial sphere $\mathcal{C S}$ [7]. From this point of view, it is expected that scattering amplitudes in four-dimensions can be recasted in terms of some sort of correlator in a certain two-dimensional conformal field theory. It has indeed been shown that soft theorems can be rewritten as Ward identities in a two-dimensional conformal field theory $[8,9]$ and hence, they should be somehow related to two-dimensional current algebras $[10,11]$. The conserved currents and the stress-tensor of the corresponding two-dimensional field theory has been discussed in [7, 12].

The S-matrix is usually defined with respect to plane waves. More recently, Pasterski, Shao and Strominger considered expanding the S-matrix on so called conformal primary 
wave functions. The wave functions are constructed by convoluting plane waves with bulk-to-boundary propagators in $A d S_{3}$, and thus transforms covariantly under the Mobius group [13]. Using this transformation allows one to transform Lorentz invariant scalar scattering amplitudes into Mobius covariant quantities, which can be considered as the correlation functions of some $2 \mathrm{~d}$ CFT. The proposed map is:

$$
\tilde{\mathcal{A}}\left(\Delta_{i}, w_{i}, \bar{w}_{i}\right) \equiv \prod_{i=1}^{n}\left(\int d^{2} z_{i} \frac{d y_{i}}{y_{i}^{3}} G_{\Delta}\left(y_{i}, z_{i}, \bar{z}_{i} ; w, \bar{w}\right)\right) \mathcal{A}\left(m_{j} \hat{p}_{j}\right),
$$

where we have $n$ copies of integration over $\mathrm{AdS}_{3}$ coordinates, and $G_{\Delta}$ are the bulk to boundary propagators. The $\mathrm{AdS}_{3}$ coordinates are imbedded in the four-dimensional momenta satisfying the massive on-shell constraint $p^{2}=-m^{2}$. Explicit results were obtained for the three-point function of $\phi^{2} \varphi$ theory, which is fixed by symmetries.

In this note we intend to explore this relation further by studying the convolution of factorisation singularities of flat space scattering amplitudes that admit a cubic diagram expansion. By ensuring that the mass is conserved at each vertex, we show that the factorisation singularity for massive poles, enforces that the $n$-point kinematics can be mapped to a configuration of a contact $\mathrm{AdS}_{3}$ Witten diagram. Once dressed with bulk to boundary propagators and integrate over the whole AdS space, one reproduce an $n$-point correlation function, i.e.:

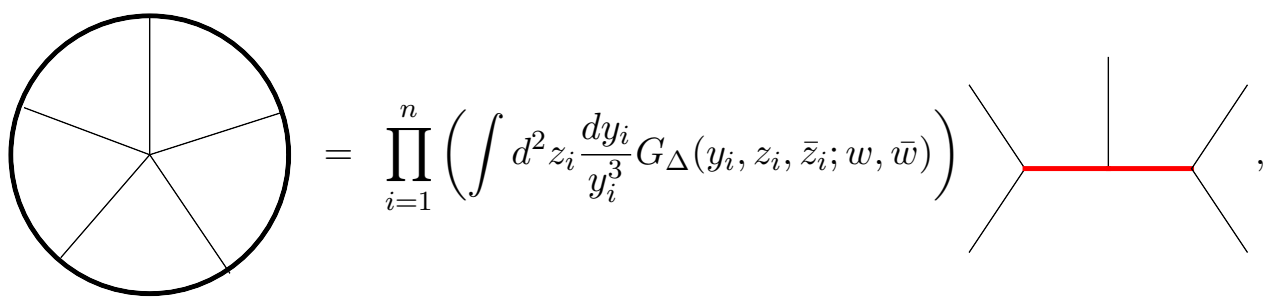

where for the cubic diagram on the r.h.s. we identify the leading singularity, which refers to when all propagators are on-shell. Since all tree-level scalar Witten diagrams can be reexpressed in terms of finite sums of contact diagrams [14-16], with the scaling dimensions of external operators being shifted, thus any Witten diagram can be rewritten as a combination of leading singularities of the massive S-matrix, dressed with appropriate conformal primary wave functions. On the other hand, if the internal propagator is massless, the leading singularity then converts to exchange Witten diagrams in the split representation.

Note that one motivation for understanding the factorisation singularity of the S-matrix under the map eq. (1.1), is if the relation between flat space S-matrix and correlation functions holds, then it is important to understand how flat space unitarity appears in the correlation function. Since we've shown that the flat space massive singularity yields contact Witten diagram, which yields log pieces of cross-ratios for the correlator, assuming that this is the sole source of logarithmic terms the unitarity of flat-space S-matrix then implies that the coefficient of $\log u$ must be definite positive.

Mass conservation can be interpreted as momentum conservation in one dimension higher, where the momentum is massless. The embedding of the previous construction in 
terms of a massless theory allows us to introduce the Cachazo-He-Yuan (CHY) representation for the propagator singularities. In particular, as discussed in [17], by parameterising the moduli space of $n$-punctures by a single parameter $\tau$ such that

$$
\sigma_{i}=\frac{v_{i}}{\tau} \quad i \in L, \quad \sigma_{i}=\tau u_{i} \quad i \in R .
$$

where $\sigma_{i}$ are the coordinates of the punctures and we've separated it into a left and right set, the parameter $\tau$ then encodes the pinch limit of the Riemann sphere. Moreover, in such parameterisation, one can identify that one of the scattering equation constraints becomes

$$
\delta\left(\tau^{2} F-p_{I}^{2}\right)
$$

where $p_{I}$ would be the associated momenta of the internal particle and $F$ is some $\tau$ independent polynomial. Thus by inserting a factor of $\delta(\tau)$ in the CHY integrand, we reproduce the factorisation constraint. This combined with the fact that there the CHY formula can be naturally computed from a world sheet chiral string theory [18, 19], give an interesting $2 d / 2 d$ correspondence:

$$
2 d C F T \leftrightarrow \prod_{i=1}^{n}\left(\int d^{2} z_{i} \frac{d y_{i}}{y_{i}^{3}} G_{\Delta}\left(y_{i}, z_{i}, \bar{z}_{i} ; w, \bar{w}\right)\right) \int d^{2 n} \sigma_{i} \mathrm{CHY}\left(\prod_{j} \tau_{j} \delta\left(\tau_{j}\right)\right),
$$

where $\tau_{i}$ are moduli for the degenerate limits of punctures on the Riemann sphere.

The remaining of this paper is organised as follows: in section 2 we quickly review the Pasterski-Shao-Strominger (PSS) proposal for the transformation of scattering amplitudes in flat space into correlation functions of conformal field theory at co-dimension two, then in section 3 we move to the study of the transform at the factorisation singularities of the S-matrix. Finally, in section 4 we propose a duality relation between correlation functions in two-dimensional chiral string theory in the CHY representation of the S-matrix and correlation functions from the PSS transformation.

\section{The PSS proposal}

The fact that $d$-dimensional conformal symmetry can be linearly realised as a $d+2$ dimensional Lorentz symmetry has a long history of applications that dates back to Dirac (see [20] for references and review). It is then natural to ask whether or not observables on both sides of the relation can also be mapped. For CFTs, the natural physical observables are correlation functions, which transform covariantly under conformal transformations. On the $d+2$-dimensional side, the natural Lorentz covariant physical observable is the S-matrix, which upon Lorentz transformation generates a little transformation. The next task is then to find a map between the variables on the two sides.

Recently Pasterski, Shao and Strominger (PSS) [13] presented a proposal for such a map, where as a first step, the four-dimensional massive momenta $\mathbb{R}^{1,3}$ is mapped into the coordinates of hyperbolic space $H_{3}$. Taking the metric of $H_{3}$ as,

$$
d s_{H_{3}}^{2}=\frac{d y^{2}+d z d \bar{z}}{y^{2}}
$$


the three-dimensional hyperbolic space is mapped to the four-dimensional momenta in a $\mathrm{SL}(2, \mathbb{C})$ covariant form as:

$$
\hat{p}_{a \dot{a}}(y, z) \equiv \hat{p}^{\mu}(y, z) \sigma_{\mu}=\frac{i}{y}\left[\begin{array}{cc}
1 & \bar{z} \\
z & y^{2}+|z|^{2}
\end{array}\right]
$$

such that $\hat{p}^{2}=\operatorname{Det}\left[\hat{p}_{a \dot{a}}\right]=-1$. For a particle of mass $m$, it's momenta is given as $p=m \hat{p}$.

It was conjectured that the correlation function of some $2 \mathrm{D}$ CFT can be related to the S-matrix in four-dimensions, where the external states are conformal primary wavefunctions, defined as:

$$
\phi_{\Delta, m}^{ \pm}\left(X^{\mu} ; w, \bar{w}\right)=\int_{0}^{\infty} \frac{d y}{y^{3}} \int d z d \bar{z} G_{\Delta}(y, z, \bar{z} ; w, \bar{w}) \exp \left[ \pm i m \hat{p}^{\mu}(y, z, \bar{z}) X_{\mu}\right]
$$

where the \pm in the exponent indicates the incoming and outgoing states and $G_{\Delta}(y, z, \bar{z} ; w, \bar{w})$ is the scalar bulk-to-boundary propagator in $H_{3}$ of conformal dimension $\Delta[21]$

$$
G_{\Delta}(y, z, \bar{z} ; w, \bar{w})=\left(\frac{y}{y^{2}+|z-w|^{2}}\right)^{\Delta}
$$

Note that we can write the bulk to boundary propagator as

$$
G_{\Delta}(y, z ; w)=\left(-i[w 1]^{a} \hat{p}(y,-z)_{a, \dot{a}}\left[\begin{array}{c}
\bar{w} \\
1
\end{array}\right]^{\dot{a}}\right)^{-\Delta}
$$

which manifests it's covariant property under the $\mathrm{SL}(2, \mathbb{C})$ transformation $w^{\prime}=(a w+$ $b) /(c w+d)$,

$$
G_{\Delta}\left(y^{\prime}, z^{\prime}, \bar{z}^{\prime} ; w^{\prime}, \bar{w}^{\prime}\right)=|c w+d|^{2 \Delta} G_{\Delta}(y, z, \bar{z} ; w, \bar{w}) .
$$

The correlation function $\tilde{\mathcal{A}}_{\Delta_{1}, \cdots, \Delta_{n}}\left(w_{i}, \bar{w}_{i}\right)$ is then related to the flat space S-matrix as defined as $\mathcal{A}(m \hat{p})$ by: ${ }^{1}$

$$
\tilde{\mathcal{A}}\left(\Delta_{i}, w_{i}, \bar{w}_{i}\right) \equiv \int d^{4} X \prod_{i=1}^{n} \phi_{\Delta_{i}, m_{i}}^{ \pm}\left(X^{\mu} ; w_{i}, \bar{w}_{i}\right) \mathcal{A}\left(m_{j} \hat{p}_{j}\right) .
$$

The four-dimensional integral $d^{4} X$ simply produces the momentum conservation delta function. The plausibility of eq. (2.7) stems from the two sides sharing the same symmetry, as verified in [13], for the case of $\phi^{2} \varphi$ interaction, where $m_{\varphi} \sim 2 m_{\phi}$.

\footnotetext{
${ }^{1}$ Here $\mathcal{A}(m \hat{p})$ is the flat space S-matrix in momentum space that is stripped of momentum conservation delta function.
} 


\subsection{The three point contact term}

The explicit example shown in [13] is a three-point contact term, and the mass of one particle is near-extremal, i.e. it is near the sum of the other two. Here we present a brief review, since most of the details will be utilised for the $n$-point construction.

Taking the mass of the first particle $\varphi$ to be $2(1+\epsilon) m$ and the masses of the other two particles be $m$. We will take $\epsilon>0$ in order for the decay process to be physical. Evaluating the $X^{\mu}$-integral, we arrive at the following expression for the scalar three-point amplitude,

$$
\begin{aligned}
\tilde{\mathcal{A}}\left(w_{i}, \bar{w}_{i}\right)=i(2 \pi)^{4} \lambda m^{-4} & \left(\prod_{i=1}^{3} \int_{0}^{\infty} \frac{d y_{i}}{y_{i}^{3}} \int d z_{i} d \bar{z}_{i}\right) \\
& \times \prod_{i=1}^{3} G_{\Delta_{i}}\left(y_{i}, z_{i}, \bar{z}_{i} ; w_{i}, \bar{w}_{i}\right) \delta^{(4)}\left(-2(1+\epsilon) \hat{p}_{1}+\hat{p}_{2}+\hat{p}_{3}\right),
\end{aligned}
$$

where we used $-p_{1}+p_{2}+p_{3}=m\left[-2(1+\epsilon) \hat{p}_{1}+\hat{p}_{2}+\hat{p}_{3}\right]$. In general, three of the four momentum conservation delta function solves one of the momenta in terms of others, while the remaining one simply enforces

$$
p_{n}^{2}=\left(\sum_{i=1}^{n-1} p_{i}\right)^{2}=-m_{n}^{2}
$$

For the current case the integral of $\left(y_{3}, z_{3}\right)$ is straightforwardly localized, leaving a Jacobian factor of

$$
-\left(-2(1+\epsilon) \frac{y_{1}^{2}+\left|z_{1}\right|^{2}}{y_{1}}+\frac{y_{2}^{2}+\left|z_{2}\right|^{2}}{y_{2}}\right)^{-1}
$$

and we are left with the final delta function, which is proportional to the on-shell condition,

$$
\delta\left(2(1+\epsilon) \frac{1}{y_{1}}-\frac{1}{y_{2}}-\frac{1}{y_{3}}\right)=\left(-2(1+\epsilon) \frac{y_{1}^{2}+\left|z_{1}\right|^{2}}{y_{1}}+\frac{y_{2}^{2}+\left|z_{2}\right|^{2}}{y_{2}}\right) \delta\left(\hat{p}_{1} \cdot \hat{p}_{2}+(1+\epsilon)\right)
$$

Note that the pre factor on the r.h.s. exactly cancels the previous Jacobian factor. Thus one concludes that momentum conservation fixes the position of one leg:

$$
z_{n}=-y_{n}\left(\sum_{i} m_{i} \frac{z_{i}}{y_{i}}\right), \quad y_{n}=\frac{-\sum_{i} m_{i} \frac{y_{i}^{2}+\left|z_{i}\right|^{2}}{y_{i}}}{1+\left(\sum_{i j} \frac{m_{i} m_{j} z_{i} \bar{z}_{j}}{y_{i} y_{j}}\right)}
$$

where the summation range is $i=1, \cdots, n-1$ and appropriate additional signs for each term depending on whether it is incoming or outgoing.

It is convenient to parameterise the remaining two $\mathrm{AdS}_{3}$ points as

$$
y_{2}=y_{1}+R \cos \theta, \quad z_{2}=z_{1}+R \sin \theta e^{i \phi},
$$

for which the argument of the delta function becomes

$$
\delta\left(\hat{p}_{1} \cdot \hat{p}_{2}+(1+\epsilon)\right)=2 y_{1}\left(y_{1}+R \cos \theta\right) \delta\left(2 \epsilon y_{1} y_{2}-R^{2}\right) .
$$




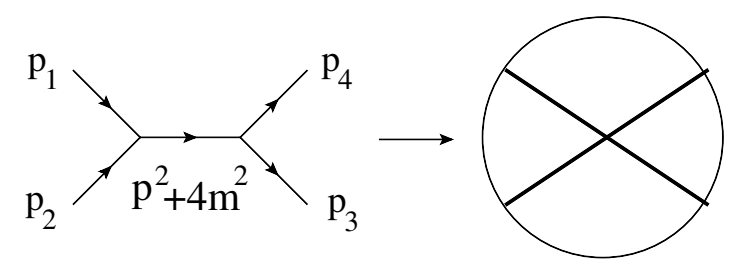

Figure 1. The factorization singularity of $s$-channel diagram leads to a contact Witten diagram in $\mathrm{AdS}_{3}$.

Thus the final constraint forces $\left(y_{2}, z_{2}\right) \rightarrow\left(y_{1}, z_{1}\right)$, and through eq. (2.11), so is $\left(y_{3}, z_{3}\right)$, arriving at a contact diagram in $\mathrm{AdS}_{3}$. Note that if $\epsilon<0$, there will be no solution for the delta function since it is below the production threshold.

The support of the delta function in equation (2.13), at leading order in $\epsilon$, is given by,

$$
R=\sqrt{2 \epsilon} y_{1}=\tilde{R} \sqrt{\epsilon}
$$

where $\tilde{R}=y_{1} \sqrt{2}$. Therefore, the integration measure translate into the coordinate $(\tilde{R}, \theta, \phi)$ as,

$$
\int d^{2} z_{1} d y_{1} \rightarrow \epsilon^{3 / 2} 2 \pi \int \tilde{R}^{2} d \tilde{R} d \Omega
$$

So more precisely, we are retaining the leading order in small $\epsilon$ expansion. In the following sections we are going to use heavily the condition (2.13), therefore the final transformation should be understood as the leading piece in $\epsilon$. More explicitly for the three-point function considered in this section we write,

$$
\begin{aligned}
\tilde{\mathcal{A}}_{3}\left(w_{i}, \bar{w}_{i}\right) \approx & 2 i \frac{(2 \pi)^{5}}{m^{4}} \lambda \epsilon^{1 / 2} \int_{0}^{\infty} \frac{d y_{2}}{\left(y_{2}\right)^{4}} \int d^{2} z_{2} \\
& \times \int \tilde{R}^{2} d \tilde{R} \int d \Omega \delta\left(\tilde{R}^{2}-2 y_{2}^{2}\right) \prod_{i=1}^{3} G_{\Delta_{i}}\left(y_{i}, z_{i}, \bar{z}_{i} ; w_{i}, \bar{w}_{i}\right) \\
\sim & \lambda \frac{\epsilon^{1 / 2}}{m^{4}} \int_{0}^{\infty} \frac{d y_{2}}{y_{2}^{3}} \int d^{2} z_{2} \prod_{i=1}^{3} G_{\Delta_{i}}\left(y_{i}, z_{i}, \bar{z}_{i} ; w_{i}, \bar{w}_{i}\right) .
\end{aligned}
$$

\section{Contact diagrams from factorization singularities}

As mentioned previously, an interesting question one can immediately pose for such a correspondence is how consistency conditions are mapped. The S-matrix is subject to locality and unitarity constraints which translate into the presence of propagator singularities as well as consistent residues. Here we will explore the implication of factorisation singularities on the $n$-copy AdS integral.

\subsection{A four-point example}

Let's assume that $(1,2)$ are incoming and $(3,4)$ are outgoing with identical absolute mass. Mass conservation restricts the $s$-channel pole to be massive. The contribution of the 
$s$-channel diagram yields:

$$
\frac{1}{\hat{p}_{1} \cdot \hat{p}_{2}+1+\epsilon}=\frac{2 y_{1} y_{2}}{\left(2 \epsilon y_{1} y_{2}-R_{12}^{2}\right)} .
$$

where we've applied a change of variable:

$$
\begin{aligned}
& y_{12}=R_{12} \cos \theta_{12}, \quad z_{12}=R_{12} \sin \theta_{12} e^{i \phi_{12}} \\
& y_{34}=R_{34} \cos \theta_{34}, \quad z_{34}=R_{34} \sin \theta_{34} e^{i \phi_{34}} .
\end{aligned}
$$

where $y_{i j} \equiv y_{i}-y_{j}$ and $z_{i j} \equiv z_{i}-z_{j}$. It is clear that there is a pole on the integration over $R_{12}$ along the real axes. Here we will focus on the contribution of the "leading" singularity associated with the propagator at $R_{12}=\sqrt{2 \epsilon y_{1} y_{2}}$. In other words, we will focus on the factorisation singularity:

$$
\delta\left(\left(p_{1}+p_{2}\right)^{2}+4 m^{2}\right)=\delta\left(\hat{p}_{1} \cdot \hat{p}_{2}+1\right)=\delta\left(\hat{p}_{3} \cdot \hat{p}_{4}+1\right),
$$

where the second equality holds on the support of overall momentum conservation. As seen from the previous section whenever the AdS coordinates appear under the constraint

$$
\hat{p}_{i} \cdot \hat{p}_{j}+1=0
$$

points $i$ and $j$ will be forced to be coincidental. Thus eq. (3.3) simply forces points 1,2 and 3,4 to be coincidental respectively. Finally, three of the four momentum conservation delta function solves one of the momenta in terms of others, and the remaining constraint is (2.9), which in this case leads to:

$$
\hat{p}_{1} \cdot \hat{p}_{3}+1=0
$$

Therefore momentum conservation forces all points to be coincidental. In other words, we end up with a contact term in $\mathrm{AdS}_{3}$ as indicated in figure 1.

Let us put all the pieces together in this simple example, to illustrate how this will work in general. From equation (2.7) for four-particles we have,

$$
\begin{aligned}
\tilde{\mathcal{A}}_{4}\left(w_{i}, \bar{w}_{i}\right)= & i(2 \pi)^{4} \frac{\lambda^{2}}{4 m^{4}}\left(\prod_{i=1}^{3} \int_{0}^{\infty} \frac{d y_{i}}{y_{i}^{3}} \int d z_{i} d \bar{z}_{i}\right) \\
& \times \prod_{i=1}^{4} G_{\Delta_{i}}\left(y_{i}, z_{i}, \bar{z}_{i} ; w_{i}, \bar{w}_{i}\right) \delta\left(\hat{p}_{1} \cdot \hat{p}_{2}+1+\epsilon\right) \delta\left(\hat{p}_{1} \cdot \hat{p}_{3}+1\right),
\end{aligned}
$$

where should be understood that $p_{4}$ has been fixed by momentum conservation, the first delta function in the second line comes from the singularity pole (3.3) whereas the second delta function comes from the remaining delta from momentum conservation (2.9), namely $\delta\left(p_{4}^{2}+m^{2}\right)$. From the explicit computation for the vertex (2.16), we have also learned that upon integration, every localisation delta function $\delta\left(\hat{p}_{i} \cdot \hat{p}_{j}+1+\epsilon\right)$, give us a leading scaling $\epsilon^{1 / 2}$, so up to a numerical factor and at leading order in $\epsilon$ we find,

$$
\tilde{\mathcal{A}}_{4}\left(w_{i}, \bar{w}_{i}\right) \sim \lambda^{2} \frac{\epsilon^{1 / 2}}{m^{4}} \int_{0}^{\infty} \frac{d y_{1}}{y_{1}^{3}} \int d^{2} z_{1} \prod_{i=1}^{4} G_{\Delta_{i}}\left(y_{i}, z_{i}, \bar{z}_{i} ; w_{i}, \bar{w}_{i}\right) .
$$


This is the contact four-point Witten diagram in $A d S_{3}$. Taking for simplicity $\Delta_{i}=\Delta, i=$ $1 \cdots 4$ and using Feynman parametrisation this integral can be done, resulting in [22, 23],

$$
\tilde{\mathcal{A}}_{4}\left(w_{i}, \bar{w}_{i}\right) \sim \lambda^{2} \frac{\epsilon^{1 / 2}}{m^{4}} \frac{\Gamma(2 \Delta-2)}{\Gamma(2 \Delta)} \frac{2 \pi^{2}}{\left(\prod_{i<j} x_{i j}\right)^{\frac{2}{3} \Delta}} F(u, v)
$$

where $F$ is a function of the cross ratios $u, v$,

$$
u=\frac{w_{12} w_{34}}{w_{13} w_{24}}, \quad v=\frac{w_{12} w_{34}}{w_{14} w_{23}}
$$

and is given by,

$$
F(u, v)=\int_{0}^{\infty} \frac{d z}{(u v)^{\frac{2}{3} \Delta}}{ }_{2} F_{1}\left(\Delta, \Delta|2 \Delta| 1-\frac{(u+v)^{2}}{(u v)^{2}}-\frac{4}{(u v)} \sinh ^{2}(z)\right) .
$$

As we see, the transformed amplitude (3.8) has the right form of a four-point correlation function in a conformal field theory. However, to explore it further, it is convenient to rewrite it in a more illuminating way in the Mellin representation. The Mellin representation of contact Witten diagram is well understood and is given as $[16],{ }^{2}$

$$
F(u, v)=\frac{1}{(2 \pi i)^{2}} \int_{-i \infty}^{i \infty} d s d t u^{s+t} v^{-t} \Gamma(-s)^{2} \Gamma(-t)^{2} \Gamma(s+t)^{2} .
$$

The above representation should be understood as a contour integral in $s$ and $t$ along the imaginary line, picking the residues at the poles of the gamma functions. It is immediately noticed that the integrand contains double poles whenever $s$ or $t$ or $(-s-t)$ are positive integers which will produce logarithmic singularities in $u$ and $v$. These logarithmic singularities corresponds to contributions from small anomalous dimensions since $\left|w_{i j}\right|^{\Delta+\gamma}=\left|w_{i j}\right|^{\Delta}\left(1+\gamma \log \left|w_{i j}\right|+\mathcal{O}\left(\gamma^{2}\right)\right)$.

Note that the fact that the factorisation singularity is connected to the single logs in the four-point function implies that if this is the sole source of logarithmic piece, then unitarity of the scattering amplitude requires that the $\log u$ piece of the correlation function must have a positive coefficient.

Even though we've only reproduced contact Witten diagrams, factorisation Witten diagrams can be represented in a similar fashion. It was shown [14-16] that the latter can be rewritten as a finite series expansion in terms of contact quartic Witten diagrams,

$$
\begin{gathered}
\sum_{k=1}^{\frac{2 \Delta_{0}-\Delta}{2}} \frac{a_{k}}{\left|w_{13}\right|^{k}} \int d p_{1} G_{k}\left(p_{1} ; w_{1}, \bar{w}_{1}\right) G_{k}\left(p_{1} ; w_{3}, \bar{w}_{3}\right) G_{\Delta_{0}}\left(p_{1} ; w_{2}, \bar{w}_{2}\right) G_{\Delta_{0}}\left(p_{1} ; w_{4}, \bar{w}_{4}\right) \\
\quad a_{k}=\frac{\left(\Delta_{0}\right)_{-k}^{2}}{4\left(\frac{2 \Delta_{0}-\Delta}{2}\right)_{1-k}\left(\frac{2 \Delta_{0}+\Delta-d}{2}\right)_{1-k}}
\end{gathered}
$$

\footnotetext{
${ }^{2}$ Notice that in two dimensions, the cross ratios $u$ and $v$ are not independent, they are related by $v=\frac{u}{1-u}$ and hence the Mellin representation (3.11) seems to be ill-defined as it should be an integration over one single variable. However, we have chosen to keep it in this form to include the more general case in higher dimensions.
} 


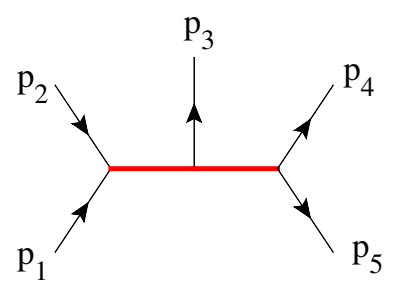

Figure 2. The five point diagram built from cubic vertices, with arrows indicating the momentum flow.

where $\Delta_{0}$ denotes the conformal dimension of the external fields, which in this note have been taken to be identical, and $\Delta$ denotes the conformal dimension of the exchanged field. Taking $w_{1}=0, w_{3}=1$, we find that factorization Witten diagrams can be identified as the residue of

$$
\mathcal{A}(\hat{p})=\sum_{k=1}^{\frac{2 \Delta_{0}-\Delta}{2}} \frac{a_{k}}{\left(\hat{p}_{1}-\hat{p}_{4}\right)} .
$$

Note that the coefficients $a_{k}$ are all positive definite which is consistent from the view point of S-matrix unitarity.

Finally, since the constraint localises all AdS points, the only possible Lorentz invariant is $\hat{p}_{i} \cdot \hat{p}_{j}=\hat{p}_{i}^{2}=-m_{i}^{2}$, i.e. the residue degenerates to a number. In other words, exchanging different higher spin states amounts to a trivial normalisation constant for the contact $\mathrm{AdS}_{3}$ diagram. ${ }^{3}$

\subsection{The $n$-point generalization}

We are now ready to make the full fledged proposal. We will consider all cubic graphs whose mass is conserved. The constraint arising from all massive propagators going onshell, along with momentum conservation at each vertex, will reduce the $n$ copies of AdS integrals to a single copy, thus corresponding to a contact Witten diagram.

Consider the five-point cubic diagram represented in figure 2, where the arrows represent the moment flow for $p_{1}+p_{2}=p_{3}+p_{4}+p_{5}$. The associated pole singularities are,

$$
\delta\left(\left(p_{1}+p_{2}\right)^{2}+4 m_{\text {in }}^{2}\right) \delta\left(\left(p_{4}+p_{5}\right)^{2}+4 m_{\text {out }}^{2}\right)
$$

where $m_{1}=m_{2}=m_{\text {in }}$ and $m_{3}=m_{4}=m_{5}=m_{\text {out }}$. This localises $\hat{p}_{1} \rightarrow \hat{p}_{2}$ and $\hat{p}_{4} \rightarrow \hat{p}_{5}$. Once again, momentum conservation can be used to solve for $\hat{p}_{3}$ and as in previous sections, the remaining on-shell condition becomes,

$$
p_{3}^{2}=-m_{\text {out }}^{2}=\left[\left(p_{1}+p_{2}\right)-\left(p_{4}+p_{5}\right)\right]^{2}=4\left(-m_{\text {in }}^{2}-m_{\text {out }}^{2}-2 m_{\text {out }} m_{\text {in }} \hat{p}_{1} \cdot \hat{p}_{3}\right) .
$$

Mass conservation ensures that $m_{\text {in }}=3 m_{\text {out }} / 2$, and one again finds,

$$
\hat{p}_{1} \cdot \hat{p}_{5}+1=0
$$

which localises $\hat{p}_{1} \rightarrow \hat{p}_{5}$, leading us to a single contact term in $A d S_{3}$.

\footnotetext{
${ }^{3}$ This can also be understood from the four-point kinematics $s$ and $t$. When $s=4 \mathrm{~m}^{2}$,

$$
t=\frac{s-4 m^{2}}{2}(1-\cos (\theta))=0
$$
}




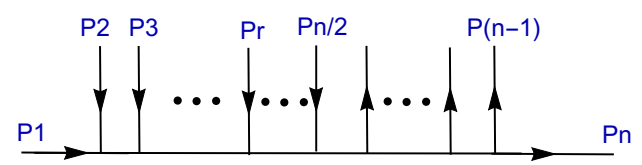

Figure 3. Half Ladder Feynman diagram.

$\boldsymbol{n}$-point ladder diagrams. The above analysis can be straightforwardly generalised to the scattering of $n$-particles for the half-ladder diagram shown in figure 3. Let us start considering an even number of particles such as, half of them are incoming with identical mass $m_{\text {in }}$ and the rest are out-going with also the same mass $m_{\text {out }}$, i.e $m_{\text {in }}=m_{\text {out }}$. Let the incoming particles to be adjacent. As before, mass conservation is imposed on each vertex. Momentum conservation allows us to fix one momentum, namely $p_{n}$. Following the order of the particle labels as in figure 3, the analysis is quite simple. The delta function singularity coming from the first propagator $\delta\left(\left(p_{1}+p_{2}\right)^{2}+4 m_{o}^{2}\right)$, forces $\hat{p}_{1} \rightarrow \hat{p}_{2}$ as we have shown before. Then the singularity from the next vertex, namely,

$$
\delta\left(\left(p_{1}+p_{2}+p_{3}\right)^{2}+9 m_{o}^{2}\right)=\delta\left(\left(2 p_{1}+p_{3}\right)^{2}+9 m_{o}^{2}\right)
$$

leads us to $\delta\left(\hat{p}_{1} \cdot \hat{p}_{3}+1\right)$, or $\hat{p}_{1} \rightarrow \hat{p}_{3}$. Continuing in this order, the singularity corresponding to the propagator at the right hand side of the vertex attached to $p_{r}$ with $r<n / 2$ is given by,

$$
\delta\left(\left(\sum_{j=1}^{r} p_{r}\right)^{2}+\left(\sum_{j=1}^{r} m_{o}\right)^{2}\right)=\delta\left(\left((r-1) p_{1}+p_{r}\right)^{2}+\left(r m_{o}\right)^{2}\right),
$$

which once again leads us to $\hat{p}_{1} \rightarrow \hat{p}_{r}$. We keep going until we reach the last propagator at the final vertex and we use it to localize the leg $p_{n-2}$ along with the previous localised $n-3$ particles at the right of it. ${ }^{4}$ Finally, the condition (2.9) plus mass conservation, leads us to

$$
p_{n}^{2}=-m_{o}^{2}=\left(\sum_{j=1}^{n / 2} p_{j}-\sum_{j=n / 2+1}^{n-1} p_{j}\right)^{2}=\left(\frac{n}{2} p_{1}-\left(\frac{n}{2}-2\right) p_{1}-p_{n-1}\right)^{2},
$$

or equivalently

$$
\hat{p}_{1} \cdot \hat{p}_{n-1}+1=0
$$

which implies $\hat{p}_{n-1} \rightarrow \hat{p}_{1}$ and therefore we end up again with a single contact term in $A d S_{3}$.

Now consider odd-number of particles, with $(n-1) / 2$ incoming and $(n+1) / 2$ outgoing. Mass conservation tells us that:

$$
m_{\text {in }}=\frac{n+1}{n-1} m_{\text {out }}
$$

and thus the only possible residue is a number.

${ }^{4}$ We can as well keep going until we reach half of the diagram at leg $n / 2$ and then perform the same analysis from right-to left until we reach the leg corresponding to particle $\frac{n}{2}+2$, in any case, we will arrive to the same conclusion. 


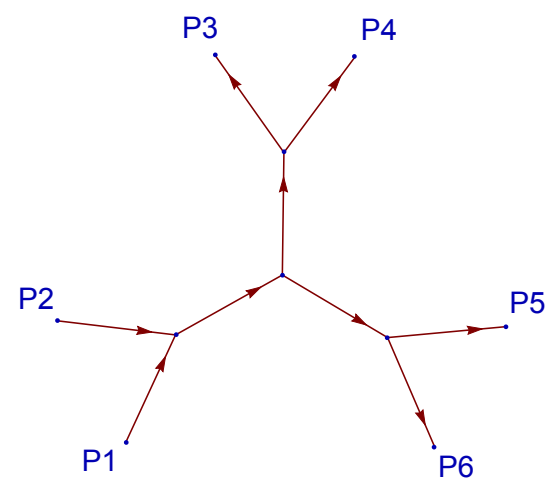

Figure 4. Benzene-type diagram.

We perform a similar procedure as before, starting from left-to-right until we reach the leg $(n-1) / 2$ and then the other way around until we reach the leg $\frac{n+1}{2}+1$. So finally the condition (2.9), leads us to

$$
p_{n}^{2}=\left(\sum_{j=1}^{(n-1) / 2} p_{j}-\sum_{j=(n+1) / 2}^{n-1} p_{j}\right)^{2}=\left(\frac{(n-1)}{2}\right)^{2}\left(p_{1}-p_{n-1}\right)^{2},
$$

which after some algebra is equivalent to

$$
\hat{p}_{1} \cdot \hat{p}_{n-1}+1=0
$$

where we have used mass conservation condition (3.18). Let us recall that on the final transform we are retaining the leading order in an $\epsilon$ expansion, and as we saw above, every delta function forcing a localisation will contribute to a $\epsilon^{1 / 2}$ scaling, therefore, the Witten contact diagram coming from the $n$-particle half-ladder appear at order $\epsilon^{(n-3) / 2}$, coming from $(n-3)$ propagators.

Benzene diagram. Now we consider the Benzene-type diagram represented in figure 4 for six-particles scattering with $p_{1}+p_{2}=p_{3}+p_{4}+p_{5}+p_{6}$. Looking at the following pole singularity,

$$
\prod_{j=\{1,3,5\}} \delta\left(\left(p_{j}+p_{j+1}\right)^{2}+4 m_{j}^{2}\right)
$$

where $m_{1}=m_{2}=m_{\text {in }}$ and $m_{3}=m_{4}=m_{5}=m_{6}=m_{\text {out }}$. It localises $\hat{p}_{j} \rightarrow \hat{p}_{j+1}$ for $j=\{1,3,5\}$. Momentum conservation can be used to solve for $p_{6}$ and the additional condition (2.9) leads us to,

$$
4 p_{6}^{2}=-4 m_{\text {out }}^{2}=\left[2 p_{1}-2 p_{3}\right]^{2}=4\left(-m_{\text {in }}^{2}-m_{\text {out }}^{2}-2 m_{\text {out }} m_{\text {in }} \hat{p}_{1} \cdot \hat{p}_{3}\right)
$$

by mass conservation we have that $m_{\text {in }}=2 m_{\text {out }}$, so, replacing it in the above equation we end up with

$$
\hat{p}_{1} \cdot \hat{p}_{3}+1=0
$$

which is again the localisation condition to $\hat{p}_{1} \rightarrow \hat{p}_{3}$. 

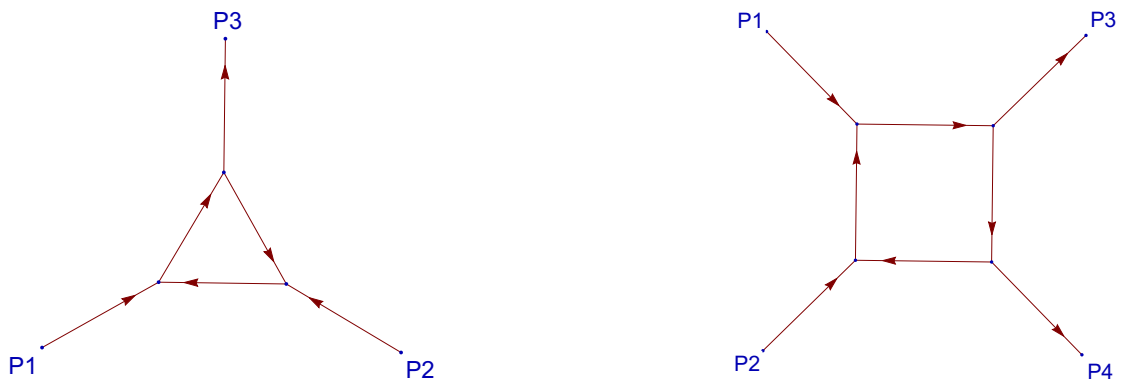

Figure 5. One-shell one-loop diagrams.

One-loop on-shell graphs. Finally, let us consider the one-loop on-shell triangle and box graphs displayed in figure 5. For the triangle, after imposing mass-conservation on every vertex, we can write the amplitude as (notice that $m_{\text {out }}=2 m_{\text {in }}$ )

$$
A\left(p_{i}\right)=\int d p_{\ell} \delta\left(p_{\ell}^{2}+m_{\ell}^{2}\right) \delta\left(\left(p_{1}+p_{\ell}\right)^{2}+\left(m_{\mathrm{in}}+m_{\ell}\right)^{2}\right) \delta\left(\left(p_{2}-p_{\ell}\right)^{2}+\left(m_{\ell}-m_{\mathrm{in}}\right)^{2}\right)
$$

The first delta function constrains the loop momentum to be on-shell, and hence the integration $d p_{\ell}$ above corresponds to a new $A d S_{3}$ point. After some algebra we can see that the second delta and third delta functions enforce the localisation of $\hat{p}_{1} \rightarrow \hat{p}_{\ell}$ and $\hat{p}_{2} \rightarrow \hat{p}_{\ell}$ respectively, and momentum conservation fix $p_{3}=p_{1}+p_{2}=2 m_{\text {in }} \hat{p}_{\ell}$.

The remaining condition from momentum conservation,

$$
p_{3}^{2}=-4 m_{\mathrm{in}}^{2}=\left(p_{1}+p_{2}\right)^{2}=-4 m_{\mathrm{in}}^{2},
$$

is satisfied by mass conservation and therefore does not impose any additional constraint.

The box is slightly more involved, but the argument applies similarly. The on-shell loop momenta condition and vertex mass conservation (taking all the external masses to be the same magnitude), allow us to write the amplitude as,

$$
\begin{aligned}
A\left(p_{i}\right)=\int d p_{\ell} \delta\left(p_{\ell}^{2}+m_{\ell}^{2}\right) \delta\left(\left(p_{1}+p_{\ell}\right)^{2}+\left(m_{\text {in }}+m_{\ell}\right)^{2}\right) \\
\quad \times \delta\left(\left(p_{1}+p_{\ell}-p_{3}\right)^{2}-m_{\ell}^{2}\right) \delta\left(\left(p_{2}-p_{\ell}\right)^{2}+\left(m_{\ell}-m_{\text {in }}\right)^{2}\right),
\end{aligned}
$$

as before, the first delta function forces the loop momenta to be on-shell, the second on the first line and the second on the second line, enforces $\hat{p}_{1} \rightarrow \hat{p}_{\ell}$ and $\hat{p}_{2} \rightarrow \hat{p}_{\ell}$ respectively. The first one on the second line can be carried out similarly leading us to,

$$
\frac{1}{\left(2 m_{\mathrm{in}}^{2}+2 m_{\mathrm{in}} m_{\ell}\right)} \delta\left(1+\hat{p}_{\ell} \cdot \hat{p}_{3}\right),
$$

hence implying $\hat{p}_{3} \rightarrow \hat{p}_{\ell}$. The remaining momentum conservation delta function reads then,

$$
p_{4}^{2}=-m_{\text {out }}^{2}=\left(p_{1}+p_{2}-p_{3}\right)^{2}=p_{1}^{2}=-m_{\text {in }}^{2},
$$

which is trivially satisfied by mass conservation. Notice that we can extend these procedure for general polygons at one-loop, i.e. beyond four-points unlike as for unitarity cuts, since the delta functions are constraining the external data instead of the loop-momentum. 
In summary, imposing the mass conservation condition coming from momentum conservation in five dimensions, plus momentum conservation in $4 \mathrm{~d}$ leads us to a Witten contact term in $A d S_{3}$ for the massive exchanges. At this point, is worth to make a couple of remarks. Contact Witten diagrams are also known as $D$-functions, as has been defined in [24], and they can be elegantly represented in terms of Mellin amplitudes [16]. So far we have considered all incoming particles to be adjacent and similarly for all the outgoing particles. This has been done in order to guarantee that we always have massive propagators, in the case where there are alternate incoming and outgoing particles, it is possible to produce massless propagators due to mass conservation. This case deserves special attention and is going to be discussed later on in this note.

\section{A 2d-2d duality}

We see that in the above construction, mass conservation plays an essential role. While this appears unnatural in a four-dimensional point of view, it arises naturally if we consider it as a five-dimensional massless $\phi^{3}$ theory, where the fifth momenta can be identified as the four-dimensional mass: $\vec{p}^{(5)}=(p, m)$. Momentum conservation in fifth dimension will then ensure that all masses are conserved at each vertex. At this point, this appears to be mere cosmetics. However, by reinterpreting the kinematics as massless, we can utilise the Cachazo, He, and Yuan (CHY) representation to reproduce the factorization singularity.

Recall that for $\phi^{3}$ theory, we can reconstruct its S-matrix by integrating over the moduli space of punctured points on the Riemann sphere [17], where the integrand is given by double cycles

$$
m^{\phi^{3}}(\alpha, \beta)=\int \prod_{a=1}^{n} \frac{d \sigma_{a}}{\operatorname{SL}(2, \mathbb{C})} \frac{1}{\left(\sigma_{\alpha_{1}, \alpha_{2}} \cdots \sigma_{\alpha_{n}, \alpha_{1}}\right)\left(\sigma_{\beta_{1}, \beta_{2}} \cdots \sigma_{\beta_{n}, \beta_{1}}\right)} \prod_{i} \delta\left(\sum_{j \neq i} \frac{s_{i j}}{\sigma_{i j}}\right),
$$

where $(\alpha, \beta)$ are any couple of permutations over the set of labels $\{1,2, \cdots, n\}, \sigma_{i}$ are the positions of the punctures on the sphere, $\sigma_{i j}=\sigma_{i}-\sigma_{j}$ and $s_{i j}=\left(p_{i}+p_{j}\right)^{2}$. By relabelling the puncture locations on the sphere as,

$$
\sigma_{a}=\frac{\tau}{u_{a}}, \quad a \in L, \quad \sigma_{a}=\frac{v_{a}}{\tau}, \quad a \in R,
$$

where $L$ and $R$ define the subset of index $L=\left\{1, \cdots, n_{L}\right\}$ and $R=\left\{n_{L}+1, \cdots, n\right\}$, it can be shown that the product of scattering equation delta functions factorises and the integrand in (4.1) takes the form:

$$
\begin{aligned}
-\left(u_{1,2} u_{1} u_{2} v_{n-1, n} v_{n-1} v_{n}\right)^{2} \frac{d \tau^{2}}{\tau^{2}} m^{\phi^{3}} & \left(\alpha_{L}, L \mid \beta_{L}, L\right)(\{u\}) \\
& \times \delta\left(p_{R}^{2}-\tau^{2} F\right) m^{\phi^{3}}\left(\alpha_{R}, R \mid \beta_{R}, R\right)(\{v\}) .
\end{aligned}
$$

Here $F$ is some $\tau$ independent polynomial, and

$$
\begin{aligned}
m^{\phi^{3}}\left(\alpha_{L}, L \mid \beta_{L}, L\right)(\{u\})= & \int \prod_{a=3}^{n_{L}} \frac{d u_{a}}{\operatorname{SL}(2, \mathbb{C})} \frac{1}{\left(u_{1} u_{1, \alpha_{L}(2)} \cdots u_{\alpha_{L}\left(n_{L}-1\right), \alpha_{L}\left(n_{L}\right)} u_{\alpha_{L}\left(n_{L}\right)}\right)} \\
& \times \frac{1}{\left(u_{1} u_{1, \beta_{L}(2)} \cdots u_{\beta_{L}\left(n_{L}-1\right), \beta_{L}\left(n_{L}\right)} u_{\beta_{L}\left(n_{L}\right)}\right)} \prod_{a \in L \backslash\{1,2\}} \delta\left(f_{L}^{a}\right)
\end{aligned}
$$


where $\left(\alpha_{L}, \beta_{L}\right)$ are any couple of permutations over the set of labels $L \backslash\{1\}$ and $f_{a}^{L}$ are the scattering equations for the subset of punctures in $L$,

$$
f_{a}^{L}=\sum_{b \in L \backslash a} \frac{s_{a, b}}{u_{a, b}} .
$$

with a similar expression for $m^{\phi^{3}}\left(\alpha_{R}, R \mid \beta_{R}, R\right)(\{v\})$ but for the subset of elements $R$ and variables $v_{a}$.

On the factorisation kinematics $p_{R}^{2}=0$, the delta function $\delta\left(p_{R}^{2}-\tau^{2} F\right)$ localises $\tau^{2}$ to vanish and hence correspond to the pinch limit of the Riemann sphere. Now we can easily turn this around and consider the pinch limit directly in the CHY integrand, which via the scattering equations will enforce the kinematics to be in the factorisation limit. For example, at four-points, one would have:

$$
\delta\left(s_{12}\right)=\int \frac{d \tau^{2}}{\tau^{2}} \delta\left(p_{R}^{2}-\tau^{2} F\right) \tau \delta(\tau)
$$

At higher points, one can reproduce all factorisation singularities by successively pinching the Riemann sphere, i.e. for $n$-factorisation singularities one simply performs a change of variable to make $n$-pinch parameters manifest, and insert $n$-factors of delta functions that localise these $n$ parameters. The result of the integration would simply a product of $n$ factorisation delta functions.

Finally we parameterise the five dimensional momenta using our AdS coordinates, where there is freedom in the embedding of four-dimensions. Depending on the embedding, the internal moment can be massive or massless. We will discuss the massless case in the next section. For the massive case, one finally establishes the following correspondence:

$$
2 d C F T \leftrightarrow \prod_{i=1}^{n}\left(\int d^{2} z_{i} \frac{d y_{i}}{y_{i}^{3}} G_{\Delta}\left(y_{i}, z_{i}, \bar{z}_{i} ; w, \bar{w}\right)\right) \int d^{2 n} \sigma_{i} \mathrm{CHY}\left(\prod_{j} \tau_{j} \delta\left(\tau_{j}\right)\right) .
$$

where $\tau_{j}$ are the moduli of the Riemann sphere whose zero limit correspond to pinched limit.

\section{Massless singularities}

In reducing the five-dimensional representation to four-dimensions, one also naturally recovers cases where the propagators are massless. For massless singularities the AdS points are no longer localised to a single point. Rather we recover configurations with multiple bulk points similar to the factorization Witten diagrams. We will demonstrate this with the previous four-point example.

Consider again the kinematics of the external lines in figure 1 but with factorisation in the $t$-channel. Now mass conservation implies that the internal propagator is massless, and hence

$$
\delta\left(\left(\hat{p}_{1}-\hat{p}_{4}\right)^{2}\right)=\frac{1}{2} \delta\left(\hat{p}_{1} \cdot \hat{p}_{4}+1\right)
$$

The factorisation singularity now identifies $\hat{p}_{1}$ to $\hat{p}_{4}$ and $\hat{p}_{2}$ to $\hat{p}_{3}$. However as momentum conservation is simply $\hat{p}_{1}+\hat{p}_{2}-\hat{p}_{3}-\hat{p}_{4}=0$, it is trivially satisfied in this limit, and hence 


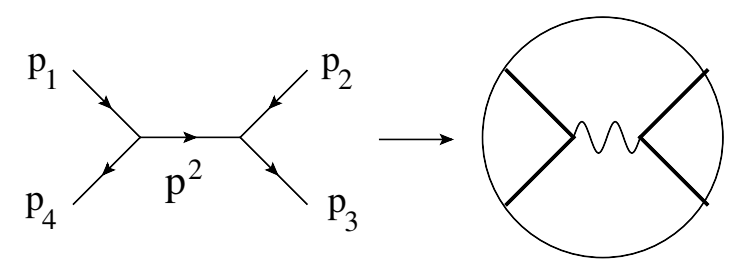

Figure 6. The factorisation singularity of $u$-channel diagram leads to a factorization Witten-like diagram in $\mathrm{AdS}_{3}$.

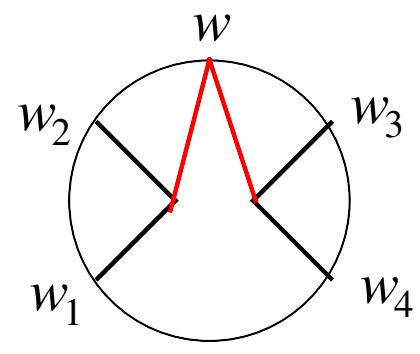

Figure 7. Schematic diagram for the massless exchange residue in $\mathrm{AdS}_{3}$.

one ends up with two free points in $\mathrm{AdS}_{3}$, i.e. putting this together we got

$\tilde{\mathcal{A}}_{4}\left(w_{i}, \bar{w}_{i}\right) \sim \lambda^{2} \frac{\epsilon^{1 / 2}}{m^{4}} \int_{0}^{\infty} \frac{d y_{1}}{y_{1}^{3}} \int d z_{1}^{2} \int_{0}^{\infty} \frac{d y_{3}}{y_{3}^{3}} \int d z_{3}^{2} \prod_{i=1}^{4} G_{\Delta_{i}}\left(y_{i}, z_{i}, \bar{z}_{i} ; w_{i}, \bar{w}_{i}\right) \delta(0) r_{t}\left(\hat{p}_{1} \cdot \hat{p}_{3}\right)$,

where $r_{t}\left(\hat{p}_{1} \cdot \hat{p}_{3}\right)$ represents the residue of the flat space amplitude around the singularity in the $t$ - channel, whose form now depends on the spin of the internal particle. This expression is a factorisation Witten-like diagram, as shown in figure 6 .

The residue of the massless singularity is now sensitive to the spin of the exchanged particle. For spin-0 the residue is simply 1, while for spin-1 one has $\hat{p}_{3} \cdot \hat{p}_{1}$. These do not appear to correspond to any bulk to bulk propagators in AdS which are non-rational functions of $\hat{p}_{3} \cdot \hat{p}_{1}$, and thus cannot correspond to any single operator exchange. We will further comment on this in the conclusions. Unlike the massive exchanges, the integral (5.2) is divergent. In order to show it, let us consider the simplest case for a residue equal to 1. By explicitly performing the integral we get [23],

$$
\tilde{\mathcal{A}}_{4}\left(w_{i}, \bar{w}_{i}\right) \sim \lambda^{2} \frac{\epsilon^{1 / 2}}{m^{4}} \frac{\pi^{2} \Gamma(\Delta-1)^{2}}{4 \Gamma(\Delta)^{2}} \frac{\delta(0)}{\left(\left|w_{12}\right|\left|w_{23}\right|\right)^{2 \Delta}}\left(\int_{0}^{\infty} \frac{d \beta}{\beta}\right)^{2} .
$$

By looking closer at the original integral (5.2), this divergence can be identified to be near the region where one or both of the integration coordinates $y_{1}, y_{2}$ are near to zero and $z_{1}$ is near to $w_{1}$ or $w_{2}$ (or $z_{2}$ is near to $w_{3}$ or $w_{3}$ ). In other words, the integral is divergent near to the boundary points $w_{i}, i=1, \cdots, 4$ and it should be regulated by an infrared cut-off. In the case of a more general residue, i.e when $r_{t}\left(\hat{p}_{1} \cdot \hat{p}_{3}\right)$ is a polynomial in $p_{1} \cdot p_{3}$, it can be seen that the degree of the divergence increases.

While the integral is divergent, it can be shown that it will be proportional to a factorised Witten diagram in the "split" representation. For any $\mathrm{SO}(3,1)$ invariant 
function $F\left(\hat{p}_{1}, \hat{p}_{2}\right)$ of two-points in AdS can be expanded in a basis of harmonic functions $\Omega$ as $[25,26],{ }^{5}$

$$
F\left(\hat{p}_{1}, \hat{p}_{2}\right)=\int_{-i \infty}^{i \infty} \frac{d c}{2 \pi i} f(c) \Omega_{c}\left(\hat{p}_{1}, \hat{p}_{2}\right)
$$

with

$$
\Omega_{c}\left(\hat{p}_{1}, \hat{p}_{2}\right)=\int_{\partial A d S} d w d \bar{w} G_{1+c}\left(\hat{p}_{1} ; w, \bar{w}\right) G_{1-c}\left(\hat{p}_{2} ; w, \bar{w}\right) .
$$

By using this representation, we can rewrite the residue $r_{t}\left(\hat{p}_{1} \cdot \hat{p}_{3}\right)$ as

$$
r_{t}\left(\hat{p}_{1} \cdot \hat{p}_{3}\right)=\int_{-i \infty}^{i \infty} \frac{d c}{2 \pi i} f_{\tilde{A}}(c) \int_{\partial A d S} d w d \bar{w} G_{1+c}\left(\hat{p}_{1} ; w, \bar{w}\right) G_{1-c}\left(\hat{p}_{3} ; w, \bar{w}\right)
$$

where

$$
f_{\tilde{A}}(c)=\frac{1}{\Omega_{c}\left(\hat{p}_{2}, \hat{p}_{2}\right)} \int d \hat{p}_{1} \Omega_{c}\left(\hat{p}_{1}, \hat{p}_{2}\right) \tilde{A}\left(p_{1} \cdot p_{3}\right)
$$

such the residue can be written as an expansion in terms of Bulk-to-Boundary propagators. By assuming we regulated the integral (5.2) by say, putting a cutoff near to the boundary, and inserting the representation (5.6) in (5.2) we can represent the massless exchange by a split diagram as in figure 7 .

\section{Conclusions and outlook}

In this note, we have considered the correspondence between four-dimensional S-matrix singularities and two dimensional CFT. This is in the context of the Pasterski, Shao and Strominger construction, where one replaces the usual S-matrix plane waves with conformal primary wave function. The kinematic space is conveniently parametrised by $\mathrm{AdS}_{3}$ coordinates, and thus correspond to $n$ - AdS bulk points. The focus on the factorisation singularities is then the first step to understand the implications of the dynamic properties of the S-matrix.

We show that for massive scalar theories, if mass conservation is implemented at each cubic vertex, the massive factorisation singularities along with over all momentum conservation, will localise the $n$-copy of AdS bulk points to a single point thus forming a contact Witten diagram. Note that in this case, the different spin exchanges simply degenerates to an overall normalisation constant. The fact that some singularities of the S-matrix are related to contact diagrams (D-functions), is somehow reminiscent to the situation in AdS/CFT where some of the singularities in the D-functions are related to S-matrix elements in flat space [27-29]. It would be interesting to pursue this connection further.

For massless singularities, the result is a factorisation Witten diagram with polynomial residues whose degree depends on the spin of the exchange particle, which are rational polynomials. However, these do not correspond to the usual bulk to bulk massless spin1 exchanges which would require non-rational functions of $\hat{p}_{i} \cdot \hat{p}_{j}$. Rather, one has an exchanged Witten diagram written in the split representation. It would be interesting to

\footnotetext{
${ }^{5}$ Here we particularise to the $A d S_{3}$ case.
} 
see if this can be rewritten in terms of a (integral) sum of usual exchange Witten diagrams with varying dimensions for the exchanged operator.

The fact that mass must be conserved at each vertex indicates that the constraints are more naturally embedded in massless constraint of five-dimensional kinematics, where the conservation is simply the extra dimensional Poincare symmetry. This identification also allows us to use the CHY representation for massless S-matrix, and reinterpret the factorization singularities as pinch limits of the Riemann sphere. This establishes an interesting $2 \mathrm{~d} / 2 \mathrm{~d}$ correspondence.

\section{Acknowledgments}

We would like to thank Shu-Heng Shao and Ellis Ye Yuan for discussions. C.C . would like to acknowledge the Mainz Institute for Theoretical Physics (MITP) for its hospitality and support during the completion of this work. Y-t Huang would also like to thank the Institute for Advanced Study for its hospitality. Y-t Huang is supported by MOST under the grant No. 103-2112-M-002-025-MY3. The work of C.C. is supported in part by the National Center for Theoretical Science (NCTS), Taiwan, Republic of China.

Open Access. This article is distributed under the terms of the Creative Commons Attribution License (CC-BY 4.0), which permits any use, distribution and reproduction in any medium, provided the original author(s) and source are credited.

\section{References}

[1] R. Penrose, Twistor algebra, J. Math. Phys. 8 (1967) 345 [InSPIRE].

[2] E. Witten, Perturbative gauge theory as a string theory in twistor space, Commun. Math. Phys. 252 (2004) 189 [hep-th/0312171] [INSPIRE].

[3] P.A.M. Dirac, Wave equations in conformal space, Annals Math. 37 (1936) 429.

[4] H. Bondi, M.G.J. van der Burg and A.W.K. Metzner, Gravitational waves in general relativity. 7. Waves from axisymmetric isolated systems, Proc. Roy. Soc. Lond. A 269 (1962) 21.

[5] R. Sachs, Asymptotic symmetries in gravitational theory, Phys. Rev. 128 (1962) 2851 [INSPIRE].

[6] S.J. Haco, S.W. Hawking, M.J. Perry and J.L. Bourjaily, The conformal BMS group, arXiv: 1701.08110 [INSPIRE].

[7] C. Cheung, A. de la Fuente and R. Sundrum, $4 D$ scattering amplitudes and asymptotic symmetries from $2 D$ CFT, JHEP 01 (2017) 112 [arXiv:1609.00732] [INSPIRE].

[8] A.E. Lipstein, Soft theorems from conformal field theory, JHEP 06 (2015) 166 [arXiv: 1504.01364] [INSPIRE].

[9] A. Strominger, Asymptotic symmetries of Yang-Mills theory, JHEP 07 (2014) 151 [arXiv: 1308.0589] [INSPIRE].

[10] T. He, P. Mitra and A. Strominger, 2D Kac-Moody symmetry of 4D Yang-Mills theory, JHEP 10 (2016) 137 [arXiv: 1503.02663] [INSPIRE]. 
[11] C. Cardona, Asymptotic symmetries of Yang-Mills with theta term and monopoles, arXiv: 1504.05542 [INSPIRE].

[12] D. Kapec, P. Mitra, A.-M. Raclariu and A. Strominger, A $2 D$ stress tensor for $4 D$ gravity, arXiv: 1609.00282 [INSPIRE].

[13] S. Pasterski, S.-H. Shao and A. Strominger, Flat space amplitudes and conformal symmetry of the celestial sphere, arXiv:1701.00049 [INSPIRE].

[14] E. D'Hoker and D.Z. Freedman, General scalar exchange in AdS(d+1), Nucl. Phys. B 550 (1999) 261 [hep-th/9811257] [inSPIRE].

[15] E. D'Hoker, D.Z. Freedman and L. Rastelli, AdS/CFT four point functions: how to succeed at $z$ integrals without really trying, Nucl. Phys. B 562 (1999) 395 [hep-th/9905049] [INSPIRE].

[16] J. Penedones, Writing CFT correlation functions as AdS scattering amplitudes, JHEP 03 (2011) 025 [arXiv: 1011.1485] [INSPIRE].

[17] F. Cachazo, S. He and E.Y. Yuan, Scattering of massless particles: scalars, gluons and gravitons, JHEP 07 (2014) 033 [arXiv:1309.0885].

[18] L. Mason and D. Skinner, Ambitwistor strings and the scattering equations, JHEP 07 (2014) 048 [arXiv: 1311.2564] [INSPIRE].

[19] Y.-t. Huang, W. Siegel and E.Y. Yuan, Factorization of chiral string amplitudes, JHEP 09 (2016) 101 [arXiv:1603.02588] [INSPIRE].

[20] W. Siegel, Embedding versus $6 D$ twistors, arXiv:1204.5679 [INSPIRE].

[21] E. Witten, Anti-de Sitter space and holography, Adv. Theor. Math. Phys. 2 (1998) 253 [hep-th/9802150] [INSPIRE].

[22] F.A. Dolan and H. Osborn, Implications of $N=1$ superconformal symmetry for chiral fields, Nucl. Phys. B 593 (2001) 599 [hep-th/0006098] [INSPIRE].

[23] W. Mueck and K.S. Viswanathan, Conformal field theory correlators from classical scalar field theory on $A d S_{d+1}$, Phys. Rev. D 58 (1998) 041901 [hep-th/9804035] [INSPIRE].

[24] E. D'Hoker et al., Graviton exchange and complete four point functions in the AdS/CFT correspondence, Nucl. Phys. B 562 (1999) 353 [hep-th/9903196] [INSPIRE].

[25] L. Cornalba, M.S. Costa and J. Penedones, Eikonal methods in AdS/CFT: BFKL pomeron at weak coupling, JHEP 06 (2008) 048 [arXiv:0801.3002] [INSPIRE].

[26] L. Cornalba, Eikonal methods in AdS/CFT: Regge theory and multi-reggeon exchange, arXiv: 0710.5480 [INSPIRE].

[27] I. Heemskerk, J. Penedones, J. Polchinski and J. Sully, Holography from conformal field theory, JHEP 10 (2009) 079 [arXiv: 0907.0151] [INSPIRE].

[28] M. Gary, S.B. Giddings and J. Penedones, Local bulk S-matrix elements and CFT singularities, Phys. Rev. D 80 (2009) 085005 [arXiv:0903.4437] [INSPIRE].

[29] J. Maldacena, D. Simmons-Duffin and A. Zhiboedov, Looking for a bulk point, JHEP 01 (2017) 013 [arXiv: 1509.03612] [INSPIRE]. 